\title{
Spring Ligament and Sustentaculum Tali Anatomical Variations: Anatomical Research Oriented to Acquired Flat Foot Study
}

\author{
by Daniel Postan $\mathrm{MD}^{1} \otimes$, Guido S. Carabelli $\mathrm{MD}^{2}$, Luciano A. Poitevin $\mathrm{MD}, \mathrm{PhD}^{3}$
}

The Foot and Ankle Online Journal 4 (9): 1

Background: Acquired flat foot is generated by the fall of the medial arch of the foot. Spring Ligament (SL) lesions and posterior tibial insufficiency have been associated with fall of medial arch. In addition, some variations of sustentaculum tali have been associated with subtalar joint osteoarthritic changes and a high risk of acquired flat foot. Anatomical variations of spring ligament have been reported. Thus, some authors observed two fascicles that composed the ligament and some observed three fascicles. The purpose of this research is to study the anatomical variations of SL and its relations with the sustentaculum tali anatomical variations to establish which fascicles of spring ligament might coincide with a sustentaculum tali-risk factor for acquired flat foot.

Materials and Methods: Fifteen feet in 10\% formalin solution were reviewed. Anatomical dissections of subtalar joint were done, and the spring ligament fascicles and sustentaculum tali variations were studied.

Results: A correlation was found between the type of sustentaculum tali and the numbers of fascicles that composed the ligament. Sustentaculum tali with two articular facets were related to spring ligaments with three fascicles, and sustentaculum tali with one articular surface were related to spring ligaments with two fascicles. The third fascicle of spring ligament was observed along with sustentaculum tali described as more stable and with less osteoarthritic changes. This observation might implicate a special role of the third fascicle in the stability of subtalar joint and the pathogenesis of acquired flat foot.

Key words: Spring Ligament, Sustentaculum Tali, Acetabulum Pedis, Acquired Flat Foot.

Accepted: August, 2011

Published: September, 2011 This is an Open Access article distributed under the terms of the Creative Commons Attribution License. It permits unrestricted use, distribution, and
reproduction in any medium, provided the original work is properly cited. @The Foot and Ankle Online Journal (www.faoj.org), 2011 All rights reserved.

T The spring ligament (SL) is an important element in supporting the medial arch of the foot.

\footnotetext{
Address correspondence to: Daniel Postan M.D. Biomechanical and Microanatomy Laboratory at $2^{\text {nd }}$ Cathedra of Anatomy. School of Medicine. Buenos Aires University,951 Uriburu Street. $2^{\text {nd }}$ Floor. Buenos Aires, Argentina. C.P. C1114AAC.

Tel.54-011-59509606 E-mail. danielpostan@hotmail.com

1 Biomechanical and Microanatomy Laboratory. $2^{\text {nd }}$ Cathedra of Anatomy. Buenos Aires University.

2 Orthopedic Surgery Division. Italian Hospital in Buenos Aires. Biomechanical and Microanatomy Laboratory. $2^{\text {nd }}$ Cathedra of Anatomy. Buenos Aires University.

${ }^{3}$ Chief Professor of Orthopedic Surgery. Buenos Aires University. Chief Professor of Anatomy. Buenos Aires University.
}

It is situated on the medial and inferior surface of the calcaneus-navicular joint. There are anatomical variations about the anatomy of spring ligament.

Although some authors observed that the SL is composed of two fascicles, there are others authors that observed that SL was composed of three fascicles. ${ }^{1-4}$
(C) The Foot and Ankle Online Journal, 2011 


\begin{tabular}{|lccc|}
\hline $\mathbf{N}$ & SLF & ST & GAP \\
\hline 6 & 2 & I & 1 \\
\hline 9 & 3 & II & 3 \\
\hline
\end{tabular}

Table 1 Results about spring ligament fascicles.

$\mathbf{N}=$ number of feet used, $\mathbf{S F L}=$ number of fascicles observed, $\mathbf{S T}=$ sustentaculum type, GAP = Gaps observed between the fascicles.

Importance of the SL in the support of medial arch is explained by its lesion, which causes the fall of medial arch. That orients the calcaneus toward valgus and forefoot toward abduction, generating an acquired flat foot. ${ }^{5-7}$

Several authors described sustentaculum tali variations, and their relation with flat foot. Thus, the calcaneus with two articular surfaces has been described as a risk factor for injuring the SL, in contradistinction to the calcaneus with three articular surfaces, which is a lesser risk factor.,

The purpose of this research is to study the anatomical variations of SL and its relations with sustentaculum talis variations to establish which fascicles of SL may coincide with a sustentaculum tali-risk factor for acquired flat foot.

\section{Materials and Methods}

Fifteen feet were fixed with $10 \%$ formalin solution. The dissections included the plantar region and the intra joint region. For the latter, the talus was disarticulated and the fibrocartilage, which covers the ligament, was dissected to visualize the fascicules of the ligament. Their arrangement, anatomical variations and the relations with posterior tibial tendon were studied.
Anatomical variations of the Sustentaculum tali were studied and linked with the SL variations. The sustentaculum tali of the calcaneus that has two articular surfaces on the talus was denominated Type II and the sustentaculum tali that has just one articular surface on the talus was denominated Type I. In this last category it is included the " 8 form" of sustentaculum tali, which has a characteristic arrangement of a horizontal number eight without the notch. The "8 form" represents a development stage halfway between both types of sustentaculum.

\section{Results}

In this research the number of fascicles of SL was variable. In nine cases we observed three fascicles of SL, and in six cases two of them. Sustentaculum tali type I was observed in six cases and type II was observed in nine cases. Sustentaculum talis that had two articular surfaces had an interfacetary notch between them. A gap was observed between either superomedial ligament or oblique ligament and the inferolateral ligament. A correlation was found between the type of sustentaculum tali and the numbers of fascicles of SL. (Table 1)

The superomedial fascicle extends from the medial portion of the sustentaculum tali to the plantar and medial portion of the navicular. It is triangular shaped with its proximal base in the calcaneus and its vertex over the navicular. It is inserted proximally in the anterior border of the sustentaculum pedis medial facet and in the medial border of the interfacetary notch, in case it is present. Distally, it inserts in the inferomedial angle of the navicular, vertically going from its medial border to the end of the fascicle in the navicular process delimiting medially the acetabulum tarsi that contains the talus. The spring complex continues medially to the upper part of the acetabulum merging with the deltoid ligament. 


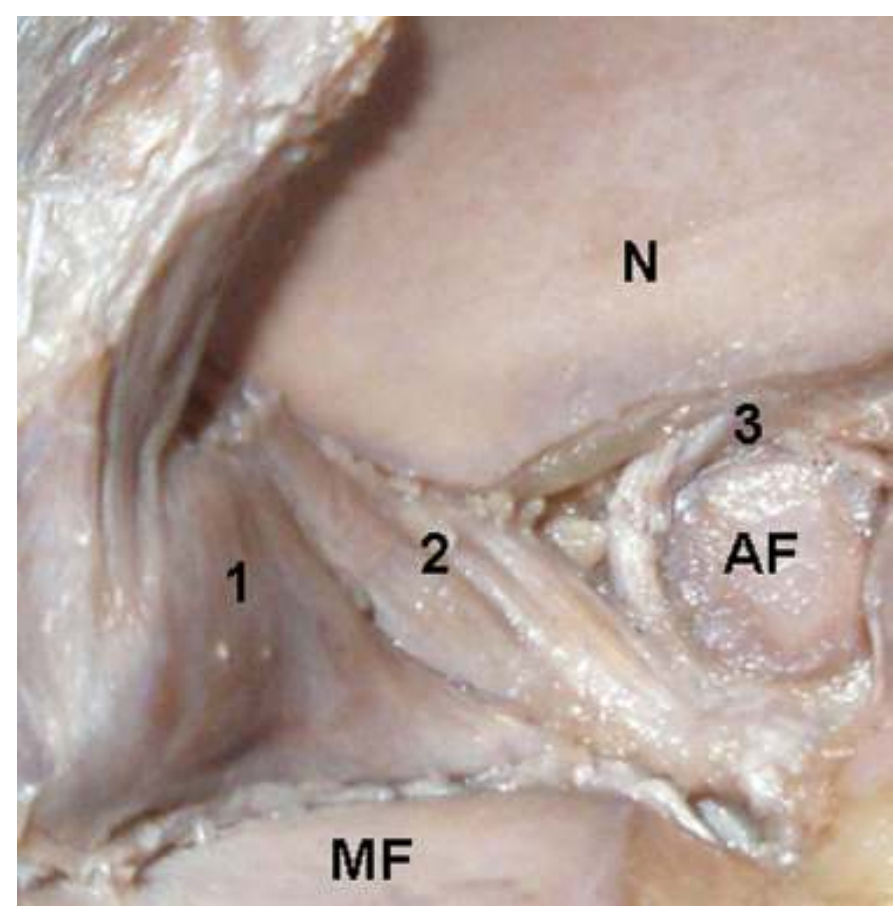

Figure 1A Type II sustentaculum tali in a right foot. The three fascicles of spring ligament can be observed. (1) Superomedial fascicle, (2) intermedium fascicle, (3) inferolateral fascicle, N Navicular Bone, MF Medial Facet, AF Anterior Facet.

Lateral to the superomedial fascicle, we observed that some fibers we termed oblique fibers that differ from the rest. These insert proximally in the interfacetary notch, when it exists, and are placed in a horizontal level, obliquely to the inferior lateral angle of the navicular articular surface. These fibers, when the interfacetary notch does not exist, are located next to the upper medial fascicle forming part thereof. We did not notice a space between the oblique fibers and the superomedial fascicle.

It is noteworthy that when the interfacetary notch existed, the oblique fibers presented a corded appearance, which differs from the superomedial fascicle, forming a third fascicle. However, in those cases in which the calcaneal facet was unique, the oblique fibers had a strip-like aspect, homogeneously continuing along the superomedial fascicle in the inferior side of the acetabulum pedis.

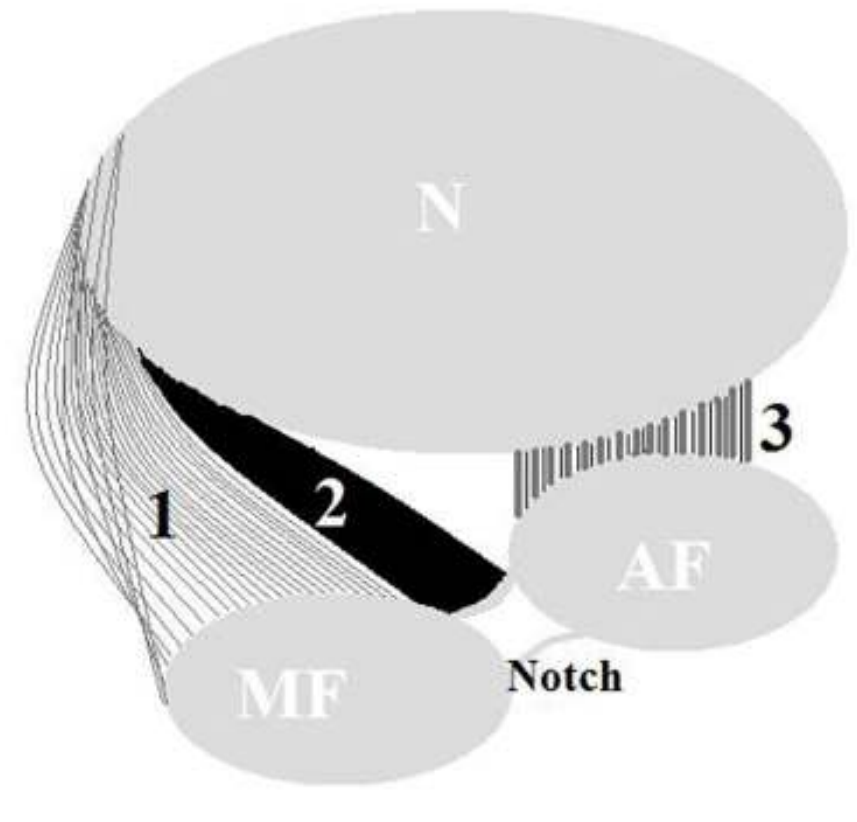

Figure 1B Outline of sustentaculum tali Type II. (See reference in Figure $1 \mathrm{~A}$ )

The inferolateral ligament goes from the anterior facet of the calcaneus or from the lateral portion of the unique facet to the navicular. Here, it inserts in its inferior edge, from the midline to the inferior external angle of the navicular. This ligament has a short course, unlike others previously described, and its anatomy can be appreciated better from the plantar side. In cases where it is inserted in the lateral portion of the interfacetary notch it demarcates in the navicular a small process in the inferior border of its articular side.

Between the oblique fibers and the inferolateral fascicle, we observe a gap in 4 of the 15 cases. It is closed by fatty tissue and it is triangular shaped, with an anterior base in the navicular and a posterior vertex oriented to the sustentaculum tali. It is placed among the insertions of the oblique fibers and the inferolateral fascicule. (Figures $1 \mathrm{AB}$ and $2 \mathrm{AB}$ ) 


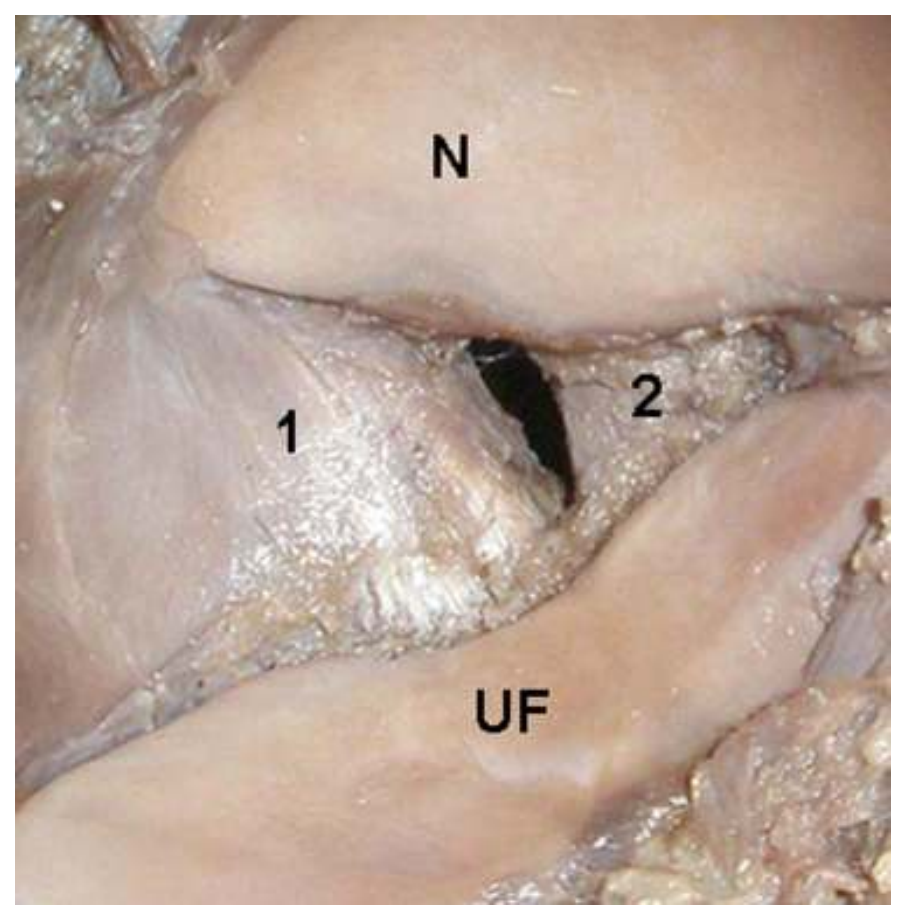

Figure 2A Type I sustentaculum tali in a right foot. A unique facet of sustentaculum tali (UF) exists in this variation. Two fascicles of SL can be seen. (1) Superomedial fascicle, (2) inferolateral, N Navicular, AF Anterior Facet, MF Medial Facet.

\section{Discussion}

The presentation of acquired flat foot can be observed in acute or insidious way. The acute presentation is thought to be induced by trauma in athletes. This may include violent inversion of the foot to ankle movement or heavy weight load through the joints.

The insidious way progresses chronically as a consequence of posterior tibial insufficiency and/or a progressive degeneration of the SL. Currently, which of the two elements trigger the pathology are still controversial and under debate.

Biomechanically, the SL participates in the static stability of the arch, together with the plantar fascia and the plantar ligaments. The dynamic stability of the arch is given by the function of the posterior tibial muscle. Normally, the posterior tibial muscle keeps the talonavicular joint rigid and fixed.

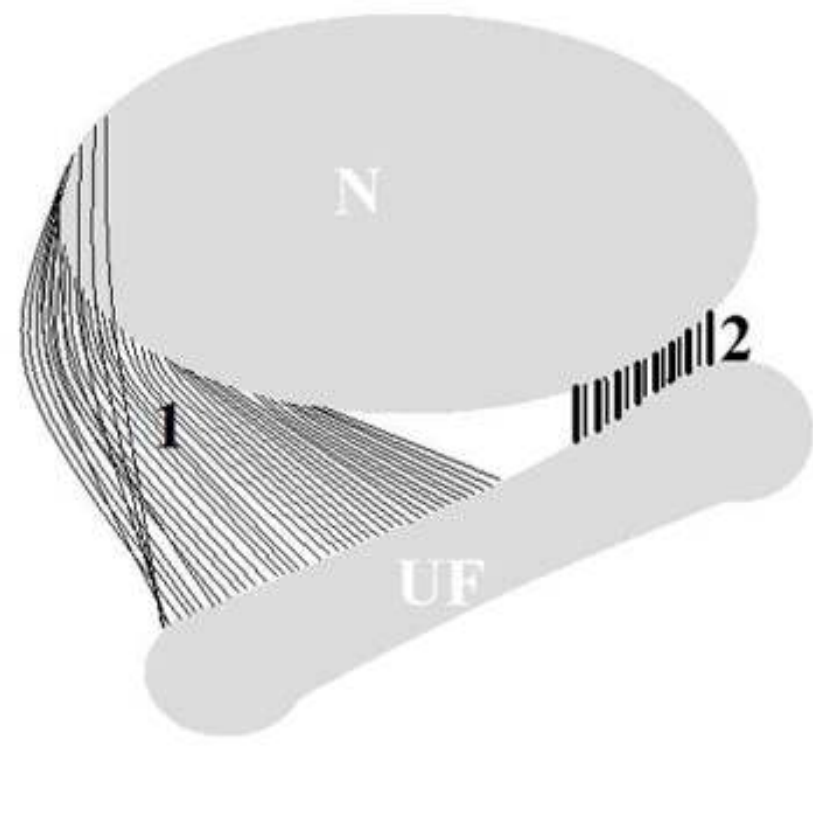

Figure 2B Outline of sustentaculum tali Type I. (See reference in Figure $2 \mathrm{~A}$ )

When walking, the lifting off the foot is seen over the metatarsals, but if the posterior tibial muscle is weak, the lifting of the foot could apply extra force to the talonavicular joint that would result in the degeneration and insufficiency of the articulation. When the medial longitudinal arch of the foot collapses, the head of the talus descends and the calcaneus everts into valgus as the forefoot abducts, forming the flat foot. ${ }^{1,5-7}$

Bruckner described a relationship between the number of articular surfaces of calcaneus and the stability of the subtalar joint. ${ }^{8}$ This author observed that the calcaneus with three articular surfaces had more stability than the calcaneus with two articular surfaces. In concordance with this hypothesis more osteoartrhitic changes have been observed in the calcaneus with type I sustentaculum tali than in calcaneus with type II. 
The SL anatomy was described in two fascicles, the superomedial and the inferolateral. ${ }^{1}$ The superomedial fascicle is triangular shaped with the base in the lateral facet of the sustentaculum tali, and vertex on the tuberosity of the navicular. On its upper part ends a fascicle of the deltoid ligament called tibio-calcaneonavicular. The inferior portion the superomedial fascicle relates with the posterior tibial tendon. Here, it has even been demonstrated the existence of synovial epithelium between both SL and posterior tibial tendon. $^{2}$ The inferolateral fascicle is strip-like formed and smaller than the superomedial one; it is placed between the lateral facet of the sustentaculum tali and the inferior border of the navicular. Between these two fascicles, a gap filled with fatty tissue was described. The intra articular portion of the SL is covered by fibrocartilage. ${ }^{1,3,4}$

In the year 2003, Taniguchi, et al., described what they called the third fascicle of the SL. ${ }^{4}$ This fascicle is located between the superomedial and the inferolateral fascicles. This author found the third fascicle in all the cases. Although in prior researches of different authors, the concept of a third SL fascicle is taken into account, however, it was not evidenced in the totality of cases., ${ }^{2,3}$

In this research, we observed that variations in the numbers of fascicles are related with the numbers of articular surfaces. Ligaments with three fascicles are related with type II sustentaculum tali, and ligaments with two fascicles are related with type I sustentaculum tali.

Given that sustentaculum tali type II is more stable than type I and has less osteoarthritic changes, is it possible to think that the third ligament (which is present more frequent in this type of sustentaculum) might be particularly involved in the subtalar joint stability? A selective biomechanical study on each fascicle is necessary to clarify this point. Despite the low number of specimens used in this research, this paper might clarify the anatomical variations of the SL complex.

\section{References}

1. Davis W, Sobel M, DiCarlo EF, Torzilli PA, Deng X, Geppert MJ, Patel M. B, Deland J. Gross, histological, and microvascular anatomy and biomechanical testing of the spring ligament complex. Foot Ankle Int 1996 17: 95-102.

2. Mengiardi B, Zanetti M, Schöttle P, Vienne B, Bode B Hodler J, Pfirrmann CWA. Spring ligament complex: MR Imaging - Anatomic correlation and findings in asymptomatic subjects. Radiology 2005 237: 242-249.

3. Patil V, Ebraheim NA, Frogameni A. Morphometric dimension of the calcaneonavicular (spring) ligament. Foot Ankle Int 2007 28: 927-931.

4. Taniguchi A, Tanaka Y, Takakura Y, Kadono K, Maeda M. Anatomy of the spring ligament. JBJS 2003 85A: 21742178.

5. Hansen ML, Otis JC, Kenneally SM, Deland JT. A closedloop cadaveric foot and ankle loading model. J Biomech 2001 34: 551-555.

6. Jennings MM, Christensen JC. The effects of sectioning the spring ligament on rearfoot stability and posterior tibial tendon efficiency. Foot Ankle Surg 2008 47: 219-224.

7. Kitaoka HB, Tae-Kun A, Luo ZP, An KN. Stability of the arch of the foot. Foot Ankle Int 199718 (10): 644-648.

8. Burckner J. Variations in the human subtalar joint. JOSTP 1987 8: 489-94.

9. Drayer-Verhagen F. Arthritis of the subtalar joint associated with sustentaculum tali facet configuration. J Anat 1993 183: 631-634.

10. Pisani G. About the pathogenesis of the so-called adult acquired pes planus. Foot ankle Surg 2010 16: 1-2.

11. Tryfonidis M, Jackson W, Mansour R, Cooke PH, Teh J, Ostlere S, Sharp RJ. Acquired adult flat foot due to isolated plantar calcaneonavicular (spring) ligament insufficiency with a normal tibialis posterior tendon. Foot Ankle Surg 2008 14: 89-95. 